
\title{
AIP $\left.\right|_{\text {Fluids of }} ^{\text {Phyic }}$
}

Stability of flow over axisymmetric bodies with porous suction strips

Ali H. Nayfeh and H. L. Reed

Citation: Physics of Fluids (1958-1988) 28, 2990 (1985); doi: 10.1063/1.865138

View online: http://dx.doi.org/10.1063/1.865138

View Table of Contents: http://scitation.aip.org/content/aip/journal/pof1/28/10?ver=pdfcov

Published by the AIP Publishing

\section{Articles you may be interested in}

Shear flow over a rotating porous plate subjected to suction or blowing

Phys. Fluids 19, 073601 (2007); 10.1063/1.2749522

Effects of body curvature and nonparallelism on the stability of flow over a swept cylinder

Phys. Fluids 6, 2363 (1994); 10.1063/1.868186

Nonlinear analysis of high Reynolds number flows over a buoyant axisymmetric body

AIP Conf. Proc. 296, 55 (1994); 10.1063/1.45292

The inviscid stability of supersonic flow past heated or cooled axisymmetric bodies

Phys. Fluids A 4, 1541 (1992); 10.1063/1.858427

Flow over bodies with suction through porous strips

Phys. Fluids 29, 2042 (1986); 10.1063/1.865590

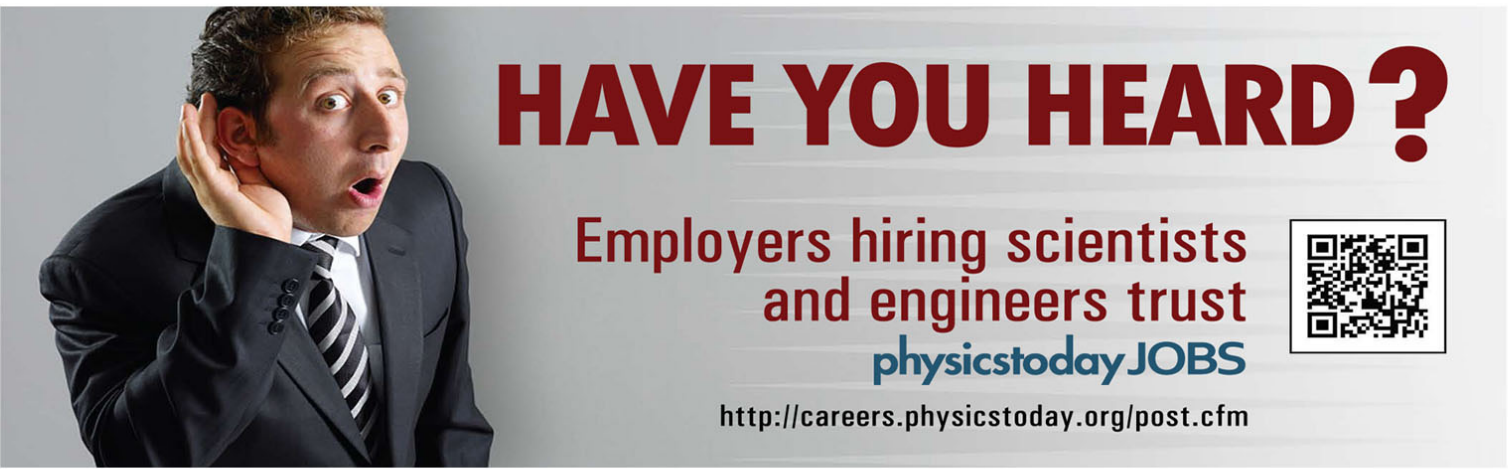




\title{
Stability of flow over axisymmetric bodies with porous suction strips
}

\author{
A. H. Nayfeh and H. L. Reed ${ }^{a)}$ \\ Department of Engineering Science and Mechanics, Virginia Polytechnic Institute and State University, \\ Blacksburg, Virginia 24061
}

(Received 11 January 1985; accepted 27 June 1985)

\begin{abstract}
A numerical-perturbation scheme is used to determine the stability of incompressible flows past axisymmetric bodies with porous strips. Linear triple deck, closed-form solutions are developed for the basic-flow quantities, which account for upstream influence. These solutions are linear superpositions of the flow past the body without suction plus the perturbations caused by the suction strips. The flow past the suctionless body is calculated using the Transition Analysis Program System (TAPS). A perturbation scheme is used to determine the increment $a_{i j}$ in the complex wavenumber at a given location $s_{j}$ because of the presence of a strip centered at $s_{i}$. The end result is a set of influence coefficients that can be used to determine the growth rates and amplification factors for any suction levels without repeating the calculations. The influence coefficients are used to develop a simple linear optimization scheme to determine the number, spacing, and mass flow rate through the strips on an axisymmetric body.
\end{abstract}

\section{INTRODUCTION}

Laminar flow control is a concept being considered for submerged, streamlined axisymmetric bodies. The use of suction through porous strips for laminar flow control is of interest in this case. To determine the effectiveness of such an approach and to optimize the number, spacing, and flow rate through such strips, one needs to determine the stability of the flow over a body with suction strips. The first step in such an approach is the calculation of the mean flow. One such available computer code is the Transition Analysis Program System (TAPS). ${ }^{1}$ This is a digital computer program designed to determine transition using the $e^{9}$ method on axisymmetric and two-dimensional bodies in air and in water. The TAPS code contains four basic components: (1) geometry, (2) potential flow, (3) boundary layer, and (4) stability analysis. The boundary layer calculations are nonsimilar and based on the Cebeci-Smith finite-difference boundarylayer program ${ }^{2}$ using the Keller box method for solving the boundary-layer equations. ${ }^{3}$ However, nonsimilar boundarylayer calculations fail to account for upstream influence. An interacting boundary-layer code would eliminate this problem, but such a code usually requires large amounts of computer storage and fails to converge for large Reynolds numbers.

For axisymmetric incompressible flows past a body with porous strips, we develop linear triple deck, closed-form solutions for the basic-flow quantities, solutions which account for upstream influence. These solutions are linear superpositions of the flow past the body without suction plus the perturbations caused by the porous suction strips. The flow past the suctionless body is calculated using the Transition Analysis Program System (TAPS). The linearized triple-deck equations are expected to be valid for strip widths $O\left(\operatorname{Re}^{-3 / 8} s_{p}^{*}\right)$ and suction levels $O\left(\operatorname{Re}^{-3 / 8} U_{\infty}^{*}\right)$ for a disturbance introduced at $s_{p}^{*}$.

\footnotetext{
a) Present address: Department of Mechanical Engineering, Stanford University, Stanford, California.
}

Using the linear triple deck, closed-form solutions, and a perturbation method, we develop a simple linear optimization scheme to determine the number, spacing, and mass flow rate through the strips on an axisymmetric body. A similar technique was developed and applied by Reed and $\mathrm{Nayfeh}^{4}$ to flows over plates with porous suction strips. The analytical results are in excellent agreement with the experimental results of Reynolds and Saric. ${ }^{5}$ In this paper we demonstrate the usefulness of our theory in predicting optimal strip distributions over axisymmetric bodies and study the effects of various adverse pressure-gradient configurations on stability.

\section{BASIC STATE}

Considering incompressible steady axisymmetric flow past a body of revolution, we define the curvilinear coordinate system $\left(s^{*}, n^{*}, \theta\right)$ shown in Fig. 1 . This is the coordinate system chosen by Mangler (see Ref. 6) and used most widely today. The surface distance from the stagnation point is $s^{*}$, the distance normal to the surface is $n^{*}$, and the circumferential angle is $\theta$. We denote the local surface radius measured from the axis as $r_{0}^{*}\left(s^{*}\right)$. All starred quantities are dimensional. We calculate the basic state as a sum of the flow (termed the mean flow) past the body without suction and the perturbations caused by the porous suction strips.

\section{A. Mean fiow}

To determine the boundary-layer equations describing the flow past the suctionless body of revolution, we define a

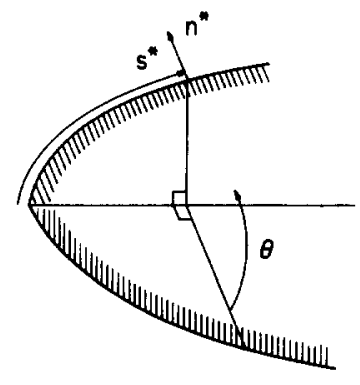

FIG. 1. Curvilinear coordinate system $\left(s^{*}, n^{*}, \theta\right)$ for a body of revolution. 
Reynolds number at a reference point $\tilde{s}^{*}$ as

$$
\operatorname{Re}=\rho^{*} U_{e^{*}}^{* s^{*}} / \mu^{*},
$$

and introduce dimensionless variables according to

$$
\begin{aligned}
& u=\frac{u^{*}}{U_{e}^{*}}, \quad v=\frac{v^{*}}{U_{e}^{*}} \sqrt{\operatorname{Re}}, \quad p=\frac{p^{*}-p_{e}^{*}}{\rho^{*} U_{e}^{* 2}}, \\
& s=\frac{s^{*}}{\bar{s}^{*}}, \quad \mathrm{n}=\left(n^{*} / \tilde{s}^{*}\right) \sqrt{\operatorname{Re}},
\end{aligned}
$$

where the subscript $e$ denotes the edge of the boundary layer, $u$ and $v$ are the velocity components tangent to and normal to the surface, respectively, and $p$ is the pressure. Assuming $r_{0}^{*}$ to be much larger than the boundary-layer thickness (i.e., the transverse-curvature effects are negligible), one finds the following boundary-layer equations governing an axisymmetric flow without suction:

$$
\begin{aligned}
& \frac{\partial}{\partial s}\left(r_{0} u\right)+r_{0} \frac{\partial v}{\partial n}=0, \\
& u \frac{\partial u}{\partial s}+v \frac{\partial u}{\partial n}=-\frac{\partial p}{\partial s}+\frac{\partial^{2} u}{\partial n^{2}}, \\
& \frac{\partial p}{\partial n}=0 .
\end{aligned}
$$

The boundary conditions are

$$
\begin{aligned}
& u=v=0 \text { at } n=0, \\
& u \rightarrow 1 \text { as } n \rightarrow \infty .
\end{aligned}
$$

To solve Eqs. (3)-(7), we need to specify the pressure distribution. In this paper we present numerical results for the case of a Rankine body ${ }^{7}$ because the edge velocity is given in closed form. Superposing a source and a sink of equal strength $4 \pi \Gamma$ in a uniform stream $U_{\infty}$, we find in the coordinate system of Fig. 2 that the velocity potential at any point $P$ is

$$
\begin{aligned}
\Phi(P)= & U_{\infty} x+\Gamma \\
& \times\left(\frac{1}{\left[(x-a)^{2}+r^{2}\right]^{1 / 2}}-\frac{1}{\left[(x+a)^{2}+r^{2}\right]^{1 / 2}}\right),
\end{aligned}
$$

where $a$ is one-half the distance between the source and the sink. Choosing $u_{r}, u_{\phi}, u_{x}$ to be the velocity components in the $r, \phi$, and $x$ directions, respectively, we find from Eq. (8) that

$$
\begin{aligned}
u_{r}= & -\Gamma \\
& \times\left(\frac{r}{\left[(x-a)^{2}+r^{2}\right]^{3 / 2}}-\frac{r}{\left[(x-a)^{2}+r^{2}\right]^{3 / 2}}\right),
\end{aligned}
$$

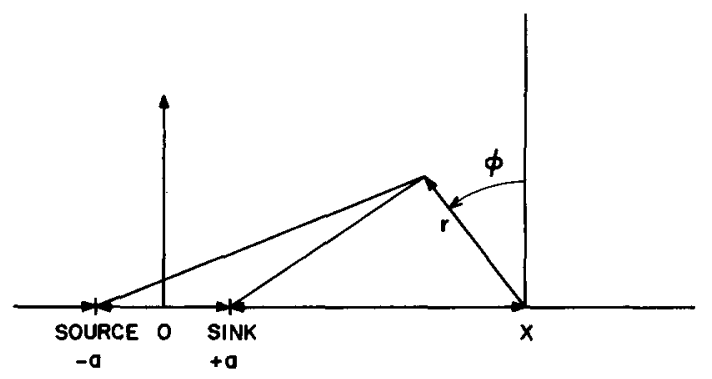

FIG. 2. Coordinate system $(r, \phi, x)$ used to describe the Rankine body.

$$
\begin{aligned}
u_{\phi}= & 0, \\
u_{x}= & U_{\infty}-\Gamma \\
& \times\left(\frac{x-a}{\left[(x-a)^{2}+r^{2}\right]^{3 / 2}}-\frac{x+a}{\left[(x+a)^{2}+r^{2}\right]^{3 / 2}}\right) .
\end{aligned}
$$

The stagnation points are then found from Eqs. (9) to be at the two coordinate locations $\left(r_{s}, x_{s}\right)$ described by

$$
\begin{aligned}
& r_{s}=0, \\
& U_{\infty}-\Gamma\left(\frac{x_{s}-a}{\left|x_{s}-a\right|^{3}}-\frac{x_{s}+a}{\left|x_{s}+a\right|^{3}}\right)=0 .
\end{aligned}
$$

In any axial plane, a streamline is described by

$$
d \psi=r u_{x} d r-r u_{r} d x .
$$

Upon integration of Eq. (11), we find that streamlines are given by

$$
\begin{aligned}
& \Gamma\left(\frac{x-a}{\left[(x-a)^{2}+r^{2}\right]^{1 / 2}}-\frac{x+a}{\left[(x+a)^{2}+r^{2}\right]^{1 / 2}}\right) \\
& \quad+\frac{1}{2} U_{\infty} r^{2}=C,
\end{aligned}
$$

where $C$ is a constant. In particular, considering Eqs. (10) we find that the stagnation streamline, the streamline describing the closed body, is given by

$$
\begin{aligned}
& \Gamma\left(\frac{x-a}{\left[(x-a)^{2}+r^{2}\right]^{1 / 2}}-\frac{x+a}{\left[(x+a)^{2}+r^{2}\right]^{1 / 2}}\right) \\
& \quad+\frac{1}{2} U_{\infty} r^{2}=0 .
\end{aligned}
$$

If we set $x=0$ in Eq. (13), we find that the maximum radius $r=r_{c}$ of the body is found by solving the relation

$$
r_{c}^{2}\left(a^{2}+r_{c}^{2}\right)^{1 / 2}=4 a \Gamma / U_{\infty} \text {. }
$$

Specifying the length of the body, the maximum radius of the body $r_{c}$, and a freestream velocity $U_{\infty}$, we can determine the source strength $4 \pi \Gamma$ and the distance between source and sink $2 a$. Knowing these quantities we immediately find the surface, or edge, speed $U_{e}$ from Eqs. (9) to be

$$
U_{e}=\left(u_{r}^{2}+u_{x}^{2}\right)^{1 / 2} \text {. }
$$

where $u_{r}$ and $u_{x}$ are given by Eqs. $(9 \mathrm{a})$ and (9c), respectively.

To generate the boundary-layer velocity profiles knowing $U_{e}$ and the geometry, we use the boundary-layer option of the Transition Analysis Program System (TAPS) ${ }^{1}$ mentioned in the introduction and obtain

$$
\begin{aligned}
& \frac{u^{*}}{U_{e}^{*}}=U_{0}(n, s), \quad \frac{v^{*}}{U_{e}^{*}}=\frac{1}{\sqrt{\operatorname{Re}}} V_{0}(n, s), \\
& \frac{P^{*}-P_{e}^{*}}{\rho^{*} U_{e}^{* 2}}=P_{0}(s) .
\end{aligned}
$$

\section{B. Linear triple deck closed-form solutions}

If a small disturbance, such as a porous strip, is introduced at $s^{*}=s_{p}^{*}$ far from the stagnation point $s^{*}=0$, then the disturbance produces a flow field that exhibits a tripledeck structure consisting of a lower deck, a main deck, and an upper deck. If the suction level is $O\left(\epsilon^{3} U_{e}^{*}\right)$, where 
$\epsilon=\operatorname{Re}_{p}^{-1 / 8}$ and $\operatorname{Re}_{p}=\rho^{*} U_{e}^{*} s_{p}^{*} / \mu^{*}$, then the effects of the disturbance occur in a neighborhood $O\left(\epsilon^{3} s_{p}^{*}\right)$ of $s_{p}^{*}$.

The lateral extent of the middle deck is $O\left(\epsilon^{4} s_{p}^{*}\right)$, which is the same as the boundary-layer thickness of the suctionless case, and thus the length scales are

$$
s^{*}=s_{p}^{*}\left(1+\epsilon^{3} X\right), \quad n^{*}=\epsilon^{4} Y_{m} x_{p}^{*} .
$$

In terms of these scales, the mean flow of Eq. (15) can be rewritten as

$$
\begin{aligned}
& u^{*} / U_{e}^{*}=U_{0}\left(Y_{m}, 1+\epsilon^{3} X\right), \\
& v^{*} / U_{e}^{*}=\epsilon^{4} V_{0}\left(Y_{m}, 1+\epsilon^{3} X\right) .
\end{aligned}
$$

Consequently, if

$$
\frac{d r_{0}}{d s} \leqslant O\left(\epsilon^{3} r_{0}\right),
$$

then the nonparallel effects of the mean flow on the triple deck structure are negligible and the flowfield has the expansions

$$
\begin{aligned}
& \frac{u^{*}}{U_{e}^{*}}=U_{0}\left(Y_{m}\right)+\epsilon \frac{d U_{0}}{d Y_{m}} A(X)+\cdots, \\
& \frac{v^{*}}{U_{e}^{*}}=-\epsilon^{2} U_{0} \frac{d A}{d X}+\cdots, \\
& \frac{p^{*}-\rho_{e}^{*}}{\rho^{*} U_{e}^{* 2}}=\epsilon^{2} p_{2}(X)+\cdots,
\end{aligned}
$$

where $U_{0}=U_{0}\left(Y_{m}, 1\right)$ is the streamwise component of the mean flow at the strip location, $A(X) \rightarrow 0$ as $X \rightarrow-\infty$, and the functions $A$ and $p_{2}$ are determined by matching the middle deck with the lower and upper decks.

The lateral extent of the upper deck is $O\left(\epsilon^{3}\right)$ and thus the lateral scale is

$$
n^{*}=\epsilon^{3} Y_{u} s_{p}^{*} .
$$

Moreover, the flowfield has the following expansions:

$$
\begin{aligned}
& u^{*} / U_{e}^{*}=1+\epsilon^{2} \hat{u}_{2}\left(X, Y_{u}\right)+\cdots, \\
& \frac{v^{*}}{U_{e}^{*}}=\epsilon^{2} \hat{v}_{2}+\cdots=-\frac{\epsilon^{2} Y_{u}}{\pi} \\
& \quad \times \int_{-\infty}^{\infty} \frac{d A(t) / d t}{(X-t)^{2}+Y_{u}^{2}} d t+\cdots, \\
& \left(p^{*}-p_{e}^{*}\right) / \rho^{*} U_{e}^{* 2}=\epsilon^{2} \hat{p}_{2}\left(X, Y_{u}\right)+\cdots,
\end{aligned}
$$

where

$$
\frac{\partial \hat{u}_{2}}{\partial X}=-\frac{\partial \hat{v}_{2}}{\partial Y_{u}} \text { and } \frac{\partial \hat{p}_{2}}{\partial Y_{u}}=-\frac{\partial \hat{v}_{2}}{\partial X} .
$$

Matching the pressures in the lower and upper decks yields

$$
p_{2}(X)=\frac{1}{\pi} \int_{-\infty}^{\infty} \frac{d A(t) / d t}{X-t} d t .
$$

The lateral extent of the lower deck is $O\left(\epsilon^{5} s_{p}^{*}\right)$ and thus the lateral scale is

$$
n^{*}=\epsilon^{5} Y_{l} s_{p}^{*} \text {. }
$$

In this deck the flowfield has the following expansions:

$$
\begin{aligned}
& u^{*} / U_{e}^{*}=\epsilon \lambda^{1 / 4} u(x, y)+\cdots, \\
& v^{*} / U_{e}^{*}=\epsilon^{3} \lambda^{3 / 4} v(x, y)+\cdots, \\
& \left(p^{*}-p_{e}^{*}\right) / \rho^{*} U_{e}^{* 2}=\epsilon^{2} \lambda^{1 / 2} p(x)+\cdots,
\end{aligned}
$$

where

$$
x=\lambda^{5 / 4} X, \quad y=\lambda^{3 / 4} Y_{l}, \quad \lambda=\frac{d U_{0}}{d Y_{m}}(0) .
$$

The functions $u, v$, and $p$ are governed by the boundary-layer equations

$$
\begin{aligned}
& \frac{\partial u}{\partial x}+\frac{\partial v}{\partial y}=0, \\
& u \frac{\partial u}{\partial x}+v \frac{\partial u}{\partial y}=-\frac{d p}{d x}+\frac{\partial^{2} u}{\partial y^{2}} .
\end{aligned}
$$

The wall boundary conditions are

$$
\begin{aligned}
& u(x, 0)=0, \\
& v(x, 0)=\left\{\begin{array}{cl}
v_{\text {wall }} & x_{\mathrm{LE}} \leqslant x \leqslant x_{\mathrm{TE}} \\
0, & \text { otherwise, }
\end{array}\right\},
\end{aligned}
$$

where $x_{\mathrm{LE}}$ and $x_{\mathrm{TE}}$ are the leading and trailing edges of the suction strip. Matching the lower and middle decks yields the boundary conditions

$$
\begin{aligned}
& u \rightarrow y+\lambda^{-3 / 4} A(x) \text { as } y \rightarrow \infty, \\
& p(x)=\frac{\lambda^{3 / 4}}{\pi} \int_{-\infty}^{\infty} \frac{d A / d t}{x-t} d t .
\end{aligned}
$$

Since $A(x) \rightarrow 0$ as $x \rightarrow-\infty$, we have the initial condition

$$
u \rightarrow y \text { as } x \rightarrow-\infty
$$

to match with the suctionless case.

The lower deck problem can be linearized for small values of $v_{\text {wall }}$. The resulting linear problem can be solved in closed form to determine $u(x, y), v(x, y)$, and $p(x)$. Then, a composite expansion valid in all decks can be obtained. Since the problem is linear, one can use superposition to determine an expansion for the case of $n$ porous strips centered at the locations $s_{i}^{*}$ and having the suction levels $v_{\text {wall }}^{*}$. We present the results here and refer the reader to Refs. 8 and 9 for the mathematical details.

For the streamwise component of velocity we have the following uniformly valid composite expansion:

$$
\frac{u^{*}}{U_{e}^{*}}\left(s^{*}, n^{*}\right)=U_{0}\left(Y_{m}\right)+\sum_{i=1}^{n} \operatorname{Re}_{i}^{1 / 4} \lambda_{i}^{-1 / 2} \frac{v_{\mathrm{wall}_{i}}^{*}}{U_{e_{i}}^{*}}\left[\left(\frac{U_{0}^{\prime}\left(Y_{m}\right)}{U_{0}^{\prime}(0)}-1\right) \bar{\delta}\left(s-s_{i}\right)+\bar{u}\left(s-s_{i}, n\right)\right],
$$

where $\lambda_{i}=U_{0}^{\prime}(0)$ at $s_{i}$ and

$$
\begin{aligned}
\bar{u}\left(s-s_{i}, n\right)= & \bar{u}_{\infty}\left[\left(\lambda_{i}^{5 / 4} / s_{i}^{*}\right)\left(s^{*} \operatorname{Re}^{3 / 8}-s_{\mathrm{LE}_{i}}^{*} \operatorname{Re}_{i}^{3 / 8}\right),\left(\operatorname{Re}_{i}^{5 / 8} / s_{i}^{*}\right) \lambda_{i}^{3 / 4} n^{*}\right] \\
& -\bar{u}_{\infty}\left[\left(\lambda_{i}^{5 / 4} / s_{i}^{*}\right)\left(s^{*} \operatorname{Re}^{3 / 8}-s_{\mathrm{TE}_{i}}^{*} \operatorname{Re}_{i}^{3 / 8}\right),\left(\operatorname{Re}_{i}^{5 / 8} / s_{i}^{*}\right) \lambda_{i}^{3 / 4} n^{*}\right], \\
\bar{\delta}\left(s-s_{i}\right)= & \bar{\delta}_{\infty}\left[\left(\lambda_{i}^{5 / 4} / s_{i}^{*}\right)\left(s^{*} \operatorname{Re}^{3 / 8}-s_{\mathrm{LE}_{i}}^{*} \operatorname{Re}_{i}^{3 / 8}\right)\right]-\bar{\delta}_{\infty}\left[\left(\lambda_{i}^{5 / 4} / s_{i}^{*}\right)\left(s^{*} \operatorname{Re}^{3 / 8}-s_{\mathrm{TE}_{i}}^{*} \operatorname{Re}_{i}^{3 / 8}\right)\right],
\end{aligned}
$$




$$
\begin{aligned}
\bar{u}_{\infty}(s, n)= & -\frac{3}{2 \pi i} \frac{|s|^{2 / 3}}{\theta^{4 / 3}}\left(\int_{0}^{\infty e^{i \pi}} \frac{e^{\tau}}{\tau^{5 / 3}} \int_{0}^{\tau^{1 / 3} n /|s|^{1 / 3}} \operatorname{Ai}(\eta) d \eta d \tau\right. \\
& +\int_{\infty}^{0} \frac{e^{\tau}}{\tau^{5 / 3}} \int_{0}^{\tau^{1 / 3} n /|s|^{1 / 3}} \operatorname{Ai}(\eta) d \eta d \tau+\frac{9}{2 \pi \theta^{2}} \int_{0}^{\infty} \frac{e^{-i \pi / 6}}{\rho^{4}+e^{I \pi / 6}} \int_{0}^{e^{i \pi / 3} \rho \theta^{1 / 3}} \operatorname{Ai}(\eta) d \eta e^{-\rho^{3} \theta|s|} d \rho \\
& \left.+\frac{9}{2 \pi \theta^{2}} \int_{0}^{\infty} \frac{e^{i \pi / 6}}{\rho^{4}+e^{-i \pi / 6}} \int_{0}^{e^{-i \pi / 3} \rho \theta^{1 / 3} n} \operatorname{Ai}(\eta) d \eta e^{-\rho^{3} \theta|s|} d \rho\right) \text { for } s>0, \\
\bar{u}_{\infty}(s, n)= & -\frac{9}{\pi \theta^{2}} \int_{0}^{\infty} \frac{\rho}{\rho^{8}+1} \int_{0}^{\rho \theta^{1 / 3} n} \operatorname{Ai}(\eta) d \eta e^{-\rho^{3} \theta|s|} d \rho \text { for } s<0, \\
\bar{\delta}_{\infty}(s)=- & \frac{|s|^{2 / 3}}{\theta^{4 / 3} \Gamma(5 / 3)}-\frac{3}{2 \pi \theta^{2}} \int_{0}^{\infty} \frac{\sqrt{3} \rho^{5}-\rho}{\rho^{8}-\sqrt{3} \rho^{4}+1} e^{-\rho^{3} \theta|s|} d \rho \text { for } s>0, \\
\bar{\delta}_{\infty}(s)=- & \frac{3}{\pi \theta^{2}} \int_{0}^{\infty} \frac{\rho}{\rho^{8}+1} e^{-\rho^{3} \theta|s|} d \rho \text { for } s<0 .
\end{aligned}
$$

Here $\mathrm{Ai}$ is Airy's function of the first kind and $\theta=\left[-3 A_{i}^{\prime}(0)\right]^{3 / 4}$. The quantities with an asterisk are dimensional. We consider $n$ porous strips, centered at $s_{1}^{*}, s_{2}^{*}, \ldots$, $s_{n}^{*}$ with the leading and trailing edges $s_{\mathbf{L E}_{i}}^{*}, s_{\mathbf{L E}_{2}}^{*}, \ldots, s_{\mathbf{L E}_{n}}^{*}$ and $s_{\mathrm{TE}_{1}}^{*}, s_{\mathrm{TE}_{2}}^{*}, \ldots, s_{\mathrm{TE}_{n}}^{*}$, respectively. We remind the reader that the $s$-Reynolds numbers at $s^{*}$ and the $i$ th strip are

$$
\operatorname{Re}=s^{*} U_{e}^{*} / v^{*}, \quad \operatorname{Re}_{i}=s_{i}^{*} U_{e i}^{*} / v_{i}^{*},
$$

respectively. The quantity $v_{\text {wall }}^{*}$ is the dimensional suction rate at the $i$ th strip. We emphasize the fact that the derivation of Eqs. (38)-(41) does not depend on Blasius mean profiles.

\section{FORMULATION OF STABILITY PROBLEM}

Referring to Fig. 1 and assuming $r_{0}^{*}$ to be much larger than the boundary-layer thickness (i.e., the transverse-curvature effects are negligible), we find that the Navier-Stokes equations in these coordinates reduce to the following:

(i) Continuity

$$
\frac{\partial u^{*}}{\partial s^{*}}+\frac{\partial v^{*}}{\partial n^{*}}=0
$$

(ii) $s^{*}$ Momentum

$$
\begin{aligned}
\rho^{*}\left(\frac{\partial u^{*}}{\partial t^{*}}+u^{*} \frac{\partial u^{*}}{\partial s^{*}}+v^{*} \frac{\partial u^{*}}{\partial n^{*}}\right) \\
=-\frac{\partial p^{*}}{\partial s^{*}}+\mu^{*}\left(\frac{\partial^{2} u^{*}}{\partial s^{* 2}}+\frac{\partial^{2} u^{*}}{\partial n^{* 2}}\right)
\end{aligned}
$$

(ii) $n^{*}$ Momentum

$$
\begin{aligned}
& \rho^{*}\left(\frac{\partial v^{*}}{\partial t^{*}}+u^{*} \frac{\partial v^{*}}{\partial s^{*}}+v^{*} \frac{\partial v^{*}}{\partial n^{*}}\right) \\
& =-\frac{\partial p^{*}}{\partial n^{*}}+\mu^{*}\left(\frac{\partial^{2} v^{*}}{\partial s^{* 2}}+\frac{\partial^{2} v^{*}}{\partial n^{* 2}}\right)
\end{aligned}
$$

To study the stability of an axisymmetric flow over a body of revolution with $n$ porous suction strips, we first define a Reynolds number $R$ as

$$
R=\rho^{*} U_{e}^{*} \delta^{*} / \mu^{*}
$$

and introduce dimensionless variables into Eqs. $(43)-(45)$ according to

$$
\begin{aligned}
& \tilde{u}=\frac{u^{*}}{U_{e}^{\star}}, \quad \tilde{v}=\frac{v^{*}}{U_{e}^{*}}, \quad \tilde{p}=\frac{p^{*}-p_{\infty}^{*}}{\rho^{*} U_{e}^{* 2}}, \\
& x=\frac{s^{*}}{\delta^{*}}, \quad y=\frac{n^{*}}{\delta^{*}}, \quad t=t^{*} \frac{U_{e}^{*}}{\delta^{\star}},
\end{aligned}
$$

where $\delta^{*}$ is the dimensional displacement thickness. In terms of these variables, the basic flow can be written as

$$
\begin{aligned}
& U=U_{0}(x, y)+\epsilon_{1} U_{1}(x, y)+\cdots, \\
& V=O\left(\epsilon_{1}^{2}\right), \\
& P=O\left(\epsilon_{1}^{2}\right),
\end{aligned}
$$

where $\epsilon_{1}=O(\epsilon)$. Consequently, to $O\left(\epsilon_{1}\right)$ the basic flow can be considered to be quasiparallel. We note that $\epsilon_{1}=O\left(R^{-1 / 4}\right)$.

We superpose small disturbances of the TollmienSchlichting form onto the basic-flow quantities to obtain total-flow quantities of the form

$$
\tilde{q}(x, y, t)=Q(x, y)+q(x, y) \exp \left(i \int k(x) d x-\omega t\right)+\cdots
$$

where $Q(x, y)$ stands for a basic-state quantity and $q(x, y, t)$ stands for a small unsteady disturbance. For the case of spatial stability, $\omega$ is real and $k$ is complex. Substituting the total-flow quantities $\tilde{u}, \tilde{v}$, and $\tilde{p}$ into the dimensionless $\mathrm{Na}$ vier-Stokes equations, subtracting the basic state, and linearizing, we obtain

$$
\begin{aligned}
& i k u+D v=0 \\
& i(k U-\omega) u+v D U=-i k p+(1 / R)\left(D^{2} u-k^{2} u\right) \\
& i(k U-\omega) v=-D p+(1 / R)\left(D^{2} v-k^{2} v\right)
\end{aligned}
$$

subject to the boundary conditions

$$
\begin{aligned}
& u=v=0 \text { at } y=0, \\
& u, v \rightarrow 0 \text { as } y \rightarrow \infty,
\end{aligned}
$$

where $D=\partial / \partial y$. We note that the problem consisting of Eqs. $(51)-(55)$ is a singular perturbation problem as $R \rightarrow \infty$ or $\epsilon_{1} \rightarrow 0$ because the small parameter multiplies the highest derivatives in Eqs. (52) and (53). Therefore, although the viscous terms proportional to $R^{-1}$ are $O\left(\epsilon_{1}^{4}\right)$, they cannot be neglected near the boundary or near the critical layer and thus they have been included in Eqs. (52) and (53).

The system of equations $(51)-(53)$ subject to the boundary conditions (54) and (55) constitutes an eigenvalue prob- 
lem for the parameters $k, \omega$, and $R$. For known basic-state velocity profiles, the equations are integrated numerically using a computer code developed by Scott and Watts ${ }^{10}$ to handle stiff two-point boundary-value problems such as this. Specifying $R$ and $\omega$, we find the eigenvalue $k$. Knowing $k$, we find the dimensional wavenumber $k^{*}=k_{r}^{*}+i k_{i}^{*}$ to be

$$
k^{*}=k / \delta^{*} \text {. }
$$

Then $-k_{i}^{*}$ is the spatial growth rate of the disturbance.

From $k_{i}^{*}$ we determine the amplification factor

$$
N=\ln \frac{A}{A_{0}}=-\int_{s_{0}^{*}}^{s^{*}} k_{i}^{*} d s^{*},
$$

where $s_{0}^{*}$ is the surface distance from the stagnation point of the location at which the constant-frequency disturbance first becomes unstable; $A$ and $A_{0}$ are the amplitudes of the disturbance at $s^{*}$ and $s_{0}^{*}$, respectively. We note that Eq. (57) is an integral over locally parallel growth rates. The $e^{N}$ method $^{11}$ cannot be used to predict the exact location of transition because of the strong dependence of transition location on freestream turbulence levels. However, linear stability theory is useful, especially for laminar flow control. It can be used as a design tool because it predicts trends for changes in the basic flow that delay transition.

\section{OPTIMIZATION SCHEME}

Taking advantage of the linearity of the triple-deck formulation, we develop a linear minimization problem which has a solution that can be used to predict efficient strip configurations. The problem is even solvable by inspection, that is, there is no need for dynamic programming such as the simplex algorithm.

We seek a first-order expansion in the form

$$
\begin{aligned}
& u=u_{0}+\epsilon_{1} u_{1}+\cdots, \\
& v=v_{0}+\epsilon_{1} v_{1}+\cdots, \\
& p=p_{0}+\epsilon_{1} p_{1}+\cdots, \\
& k=k_{0}+\epsilon_{1} k_{1}+\cdots,
\end{aligned}
$$

where the quantities with the subscript 0 represent suctionless-body disturbance quantities and those with the subscript 1 represent the contributions caused by the presence of the suction strips. To carry out the expansion given by Eqs. (58)-(61) to $O\left(\epsilon_{1}^{2}\right)$, one needs to include the normal component of velocity generated by the suction strips and the second-order term in $U$ according to Eqs. (48)-(50). To carry out the expansion to $O\left(\epsilon_{1}^{3}\right)$, one needs to include the terms up to $O\left(\epsilon_{1}^{3}\right)$ in Eqs. (48) and (49) and the nonparallel effects of the flow over the suctionless body. Application of the present first-order theory to the case of flow over flat plates with suction strips yields results that are in good agreement with the experimental results of Reynolds and Saric. ${ }^{5}$ Substituting Eqs. (58)-(61) and (48) $-(50)$ into Eqs. (51) $-(55)$ and equating coefficients of like powers of $\epsilon_{1}$, we obtain the following.

(i) Order $\epsilon_{1}^{0}$ :

$$
\begin{aligned}
L_{1}\left(u_{0}, v_{0}, p_{0}\right)= & i k_{0} u_{0}+D v_{0}=0, \\
L_{2}\left(u_{0}, v_{0}, p_{0}\right)= & -i \omega u_{0}+i k_{0} u_{0} U_{0}+v_{0} D U_{0}+i k_{0} p_{0} \\
& -R^{-1}\left(D^{2} u_{0}-k_{0}^{2} u_{0}\right)=0,
\end{aligned}
$$

$$
\begin{aligned}
& L_{3}\left(u_{0}, v_{0}, p_{0}\right)=-i \omega v_{0}+i k_{0} v_{0} U_{0}+D p_{0} \\
& \quad-R^{-1}\left(D^{2} v_{0}-k_{0}^{2} v_{0}\right)=0, \\
& u_{0}=v_{0}=0 \text { at } y=0, \\
& u_{0}, v_{0} \rightarrow 0 \text { as } y \rightarrow \infty .
\end{aligned}
$$

(ii) Order $\epsilon_{1}$ :

$$
\begin{aligned}
& L_{1}\left(u_{1}, v_{1}, p_{1}\right)=-i k_{1} u_{0}, \\
& L_{2}\left(u_{1}, v_{1}, p_{1}\right)=-i k_{1} u_{0} U_{0}-i k_{0} u_{0} U_{1}-v_{0} D U_{1} \\
&-i k_{1} p_{0}-2 R^{-1} k_{0} k_{1} u_{0}, \\
& L_{3}\left(u_{1}, v_{1}, p_{1}\right)=-i k_{1} v_{0} U_{0}-i k_{0} v_{0} U_{1}-2 R^{-1} k_{0} k_{1} v_{0}, \\
& u_{1}=v_{1}=0 \text { at } y=0, \\
& u_{1}, v_{1} \rightarrow 0 \text { as } y \rightarrow \infty .
\end{aligned}
$$

Equations (62)-(66) constitute an eigenvalue problem for $\left(\omega, k_{0}, R\right)$ and the disturbances $\left(u_{0}, v_{0}, p_{0}\right)$ superposed on an otherwise undisturbed suctionless-body boundary-layer flow. Since the homogeneous parts of Eqs. (67)-(71) are the same as Eqs. (62)-(66) and because the latter have a nontrivial solution, a solution exists for the inhomogeneous problem (67)-(71) only if a solvability condition is satisfied. ${ }^{12}$ The inhomogeneous terms on the right-hand sides must be orthogonal to every solution of the adjoint homogenous problem. That is,

$$
\begin{gathered}
k_{1} \int_{0}^{\infty}\left[i u_{0} z_{1}+i p_{0} z_{2}+\left(i U_{0}+2 k_{0} R^{-1}\right)\left(u_{0} z_{2}+v_{0} z_{3}\right)\right] d y \\
=-\int_{0}^{\infty}\left[\left(i k_{0} u_{0} U_{1}+v_{0} D U_{1}\right) z_{2}+i k_{0} v_{0} U_{1} z_{3}\right] d y,
\end{gathered}
$$

where the adjoint is defined by

$i k_{0} z_{2}-D z_{3}=0$,

$i k_{0} z_{1}+i\left(k_{0} U_{0}-\omega\right) z_{2}-R^{-1}\left(D^{2} z_{2}-k_{0}^{2} z_{2}\right)=0$,

$-D z_{1}+z_{2} D U_{0}+i\left(k_{0} U_{0}-\omega\right) z_{3}-R^{-1}$

$\times\left(D^{2} z_{3}-k_{0}^{2} z_{3}\right)=0$,

$z_{2}=z_{3}=0$ at $y=0$,

$z_{n} \rightarrow 0$ as $y \rightarrow \infty$.

At each $s$, since $k_{1}$ is independent of $\epsilon_{1}$ according to Eq. (61), the correction to $k_{0}$ is a linear function of $\epsilon_{1}$. Recalling the definition of $\epsilon_{1}$ we conclude that $\epsilon_{1} k_{1}$ is directly proportional to the suction rate.

Using the linear dependence of $\epsilon_{1} k_{1}$ on the suction rate, we consider an axisymmetric body with $n$ porous strips and $m$ specified points of computation between branch $I$ and branch II of the stability curve. We solve the zero-order eigenvalue problem to find the suctionless-body complex wavenumber $k_{B_{j}}$ and the eigenvectors $u_{0}, v_{0}$, and $p_{0}$ and their adjoints $z_{1}, z_{2}$, and $z_{3}$ at each point $j=1,2, \ldots, m$. Then, using linear triple-deck theory, we calculate the function

$$
\left[U_{0}^{\prime}(y) / U_{0}^{\prime}(0)-1\right] \bar{\delta}\left(s_{j}-s_{i}\right)+\bar{u}\left(s_{j}-s_{i}, n\right)
$$

at each point of computation $j=1,2, \ldots, m$ caused by each individual strip $i=1,2, \ldots, n$, where $\bar{u}$ and $\bar{\delta}$ are defined in Eqs. (38) and (39), respectively. Then applying Eq. (72) we determine $k_{1}$ for each $i$ and $j$ and call this quantity $a_{i j}$. Thus the total complex wavenumber $k_{j}$ at point $j$ because of the 
presence of all $n$ strips is

$$
k_{j}=k_{B_{j}}+\sum_{i=1}^{n} a_{i j} \frac{v_{\mathrm{wall}_{i}}^{*}}{U_{e i}^{*}} \operatorname{Re}_{i}^{1 / 4} \lambda_{i}^{-1 / 2} .
$$

To optimize a suction distribution keeping the total dimensional mass-flow rate constant, we minimize the amplification factor

$$
N=\ln \frac{A}{A_{0}}=-\int_{s_{0}^{*}}^{s^{*}} k_{i}^{*} d s^{*} .
$$

Although the expansion is carried out to $O\left(\epsilon_{1}\right)$ and the stability analysis is quasiparallel so that the integral in Eq. (80) is over locally parallel growth rates, application of the present theory to the case of flow over flat plates with suction strips yields $N$ factors that are in good agreement with the experimental results of Reynolds and Saric. ${ }^{5}$ Thus Eq. (80) seems to be a suitable target for optimization. Using the trapezoidal rule to perform the integration in $\mathrm{Eq}$. (80) and applying $\mathrm{Eq}$. (79), we find that

$$
\begin{aligned}
& N=-\operatorname{Im} \sum_{j=2}^{m}\left(\frac{k_{B_{j}}}{\delta_{j}^{*}}+\frac{k_{B_{j-1}}}{\delta_{j-1}^{*}}\left(s_{j}^{*}-s_{j-1}^{*}\right)\right) \\
&+\sum_{i=1}^{n} c_{i} \frac{v_{\text {wall }_{i}}^{*}}{U_{e i}^{*}}, \\
& c_{i}=-\operatorname{Im} \sum_{j=2}^{m}\left(\frac{a_{i j}}{\delta_{j}^{*}}+\frac{a_{i j-1}}{\delta_{j-1}^{*}} \operatorname{Re}_{i}^{1 / 4} \lambda_{i}^{-1 / 2}\left(s_{j}^{*}-s_{j-1}^{*}\right)\right) .
\end{aligned}
$$

Therefore, to minimize $N$, we evaluate and store the coefficients $c_{i}$ and determine the solution $v_{\text {wall }_{i}}^{*} / U_{e i}^{*}, i=1,2, \ldots, n$ for

$$
\min \left[c_{i}\left(v_{\text {wall }_{i}}^{*} / U_{e i}^{*}\right)\right],
$$

subject to constraints that give a reasonable physical picture. Such constraints involve a ceiling on $\left|v_{\text {wall }}^{*} / U_{e i}^{*}\right|$, a lower limit on $\left|v_{\mathrm{wall}_{i}^{*}}^{*} / U_{e i}^{*}\right|$, a distribution that keeps $-k_{i}^{*}$ positive after the disturbance first goes unstable, and a distribution that eliminates displacement thickness effects. Obviously, if the only constraint we impose is an upper bound $v_{u}$ on $\left|v_{\text {wall }}^{*} / U_{e i}^{*}\right|$, then Eq. (83) is satisfied by assigning the $v_{\text {wall }_{i}}^{*} /$ $U_{e i}^{*}$ and multiplying the largest $c_{i}$ by the value of $-v_{u}$, then doing the same for the next largest $c_{j}$, etc. Thus the optimization scheme predicts a concentration of the suction at the branch I neutral point as in the two-dimensional case. ${ }^{4}$

\section{RESULTS AND DISCUSSION}

In this section we first show results of our optimization scheme. We choose a Rankine body $4.88 \mathrm{~m}$ long with a maximum body radius of $0.3 \mathrm{~m}$. The freestream unit Reynolds number per meter is 6.6 million and the freestream velocity is approximately $6.4 \mathrm{~m} / \mathrm{sec}$. The body and the edge-velocity distribution are shown in Fig. 3.

We introduce a disturbance of constant dimensionless frequency $F=15.0 \times 10^{-6}$ into the boundary layer of this body. Here "dimensionless" means with respect to freestream variables, not edge variables. Figure 4 shows the amplification factor $N$ plotted versus the dimensional surface distance (from the stagnation point) between branch $I$ and

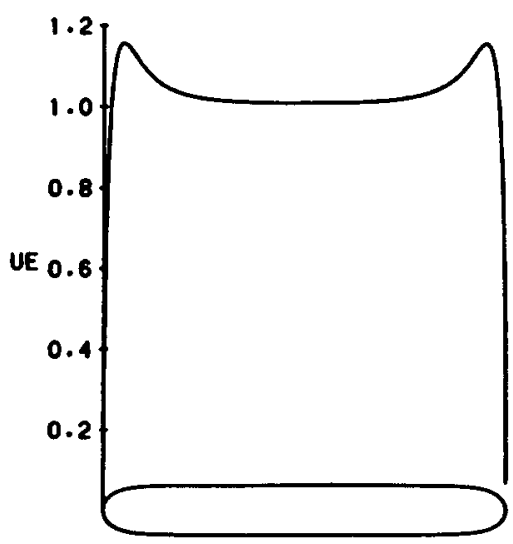

FIG. 3. Ratio of edge speed to freestream speed for a Rankine body $4.88 \mathrm{~m}$ long with a maximum body radius of $0.3 \mathrm{~m}$.

branch II of the stability curve for the suctionless body. The amplification factor attains a maximum of 40.8 at the branch II neutral point. The velocity distribution from branch I to branch II is also provided in this figure and indicates that the greatest contribution to the disturbance growth occurs near branch I where the pressure gradient is the most adverse.

Our plan is to consider a uniform distribution of suction through strips and then to try to optimize it, keeping the total dimensional mass flow rate a constant. We examine a configuration of fifteen $0.01 \mathrm{~m}$ wide strips distributed evenly between branch I and branch II. Strip centers are spaced at $0.09-\mathrm{m}$ intervals and suction levels are $-4.93 \times 10^{-4}$ times the freestream velocity. This corresponds to a volumetric flow rate per unit width of $4.73 \times 10^{-4} \mathrm{~m}^{3} / \mathrm{sec} / \mathrm{m}$ for the suction system. Using the linearized scheme discussed in Sec. IV, we calculate the growth rates between branches I and II and then the amplification factor. The amplificationfactor distribution is shown in Fig. 5. The uniform distribution of suction, itself, reduces the maximum amplification factor dramatically to a level of 16.7 . It is now our intent to reduce this level even further by redistributing the suction.

Considering the optimization scheme, we find the influence coefficients $c_{i}$ to be as given in Fig. 6. The influence

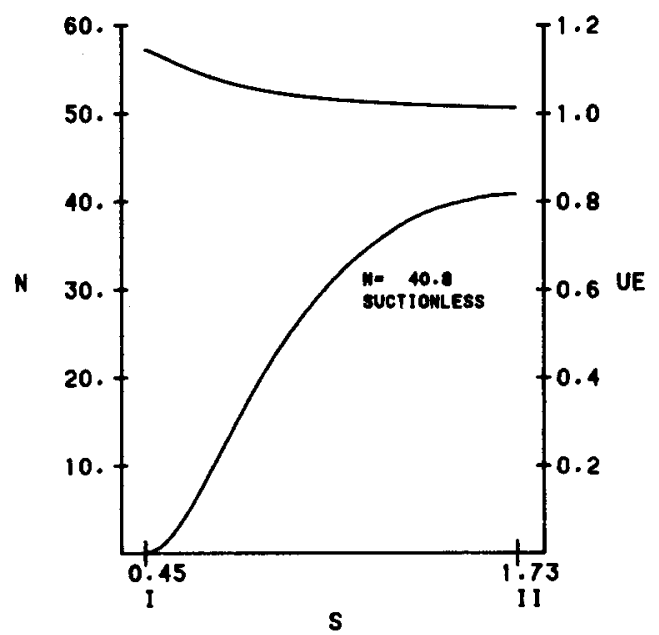

FIG. 4. Amplification-factor curve for the suctionless body, $F=15.0 \times 10^{-6}$. Edge-speed ratio shown as top curve. 


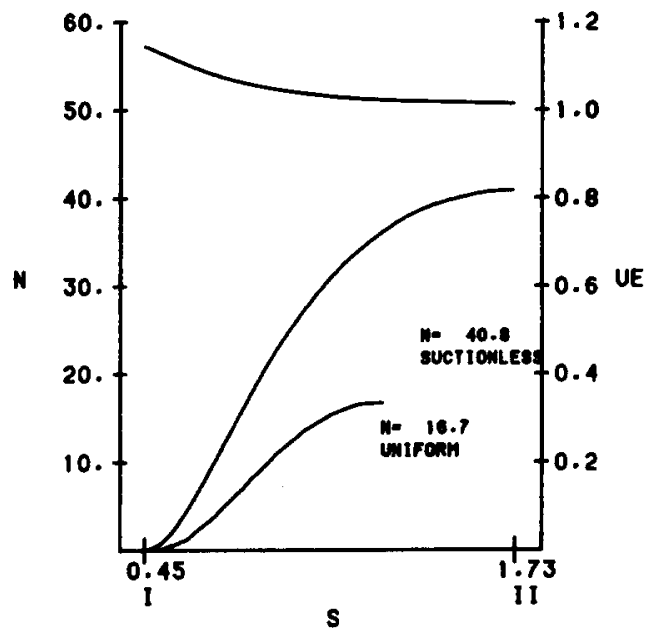

FIG. 5. Comparison of the amplification-factor curve for a suctionless case with that for the case of 15 equally spaced suction strips between branch I and branch II, $0.01 \mathrm{~m}$ wide, equal suction, $F=15.0 \times 10^{-6}$. Edge-speed ratio is shown as top curve.

coefficients for this axisymmetric problem near branch $I$ are a hundred times larger than those near branch II, in contrast to the two-dimensional problem where all coefficients are approximately the same order of magnitude. So it appears that suction concentrated in the branch I region, the region associated with the more adverse pressure gradient, is extremely effective for stabilization.

Suppose we are free to put any number of $0.01 \mathrm{~m}$ wide strips on the body but require that the minimum distance between strip centers be $0.09 \mathrm{~m}$. The linear minimization scheme with the physical constraints taken into account predicts that if we use 11 strips with optimized suction levels and spacings, then the maximum amplification factor can be reduced significantly from that of the uniform problem above to 8.0. Figure 7 compares the optimized amplificationfactor distribution with both the uniform suction and suctionless cases. The growth of the disturbance is eliminated

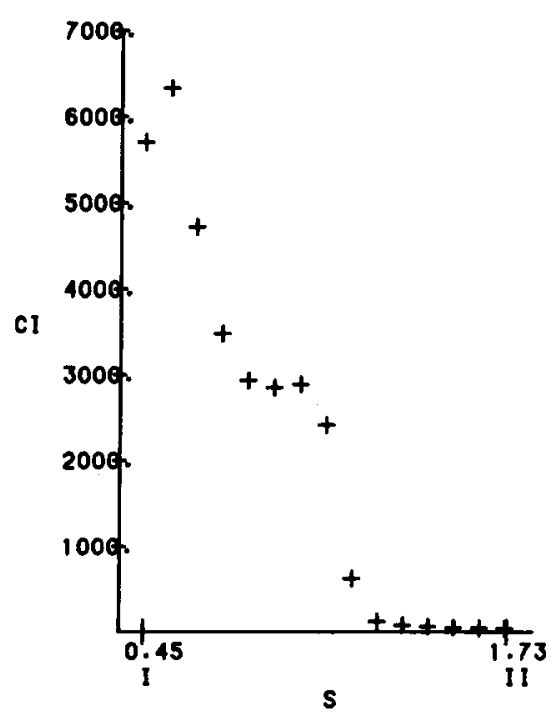

FIG. 6. Influence coefficients $c_{i}$ for an axisymmetric body.

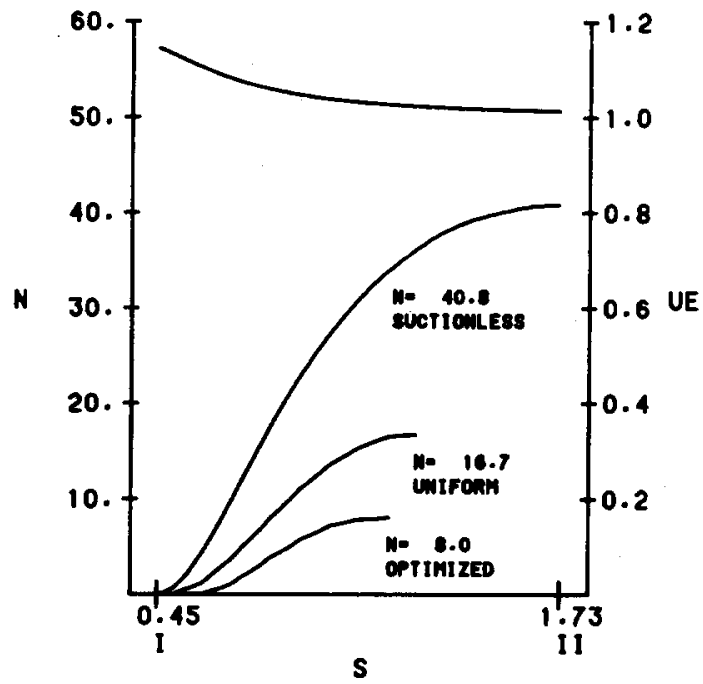

FIG. 7. Amplification-factor curve for 11 strips between branch I and branch II, $0.01 \mathrm{~m}$ wide, spacing and suction level are optimized in order to decrease $\ln \left(A / A_{0}\right), F=15.0 \times 10^{-6}$. The amplification-factor curves corresponding to the suctionless case and the case of 15 uniform equally spaced strips are shown for comparison. Edge-speed ratio is shown as top curve.

completely in the branch II region where the pressure is the least adverse. It is apparent that controlling disturbances while their growth rates are still small is desirable.

These results show the usefulness of our linear optimization scheme in predicting the suction level, spacing, and number of strips to decrease the disturbance growth as much as possible on an axisymmetric body. We have finished the development of and documentation for a computer code for official distribution that will interface with TAPS and suggest efficient configurations using our theory.

Calculations for this work were done in the adverse pressure-gradient region near the nose of the body. Deceleration of a flow is another destabilizing effect adding to the amplification factor. But we find the influence coefficients in the adverse pressure-gradient area near branch $I$ to be an

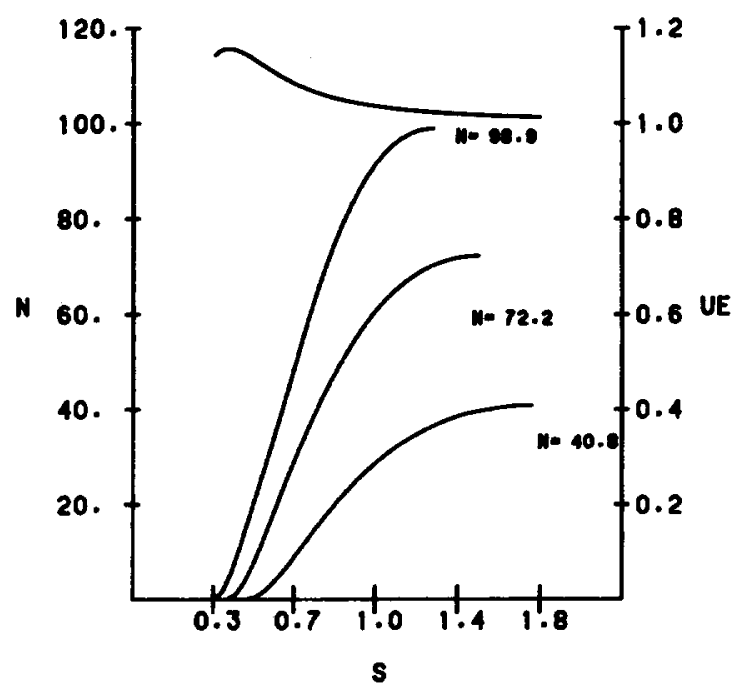

FIG. 8. Amplification-factor curves for different pressure-gradient situations on the suctionless body, $F=15.0 \times 10^{-6}$. Edge-speed ratio is shown as top curve. 


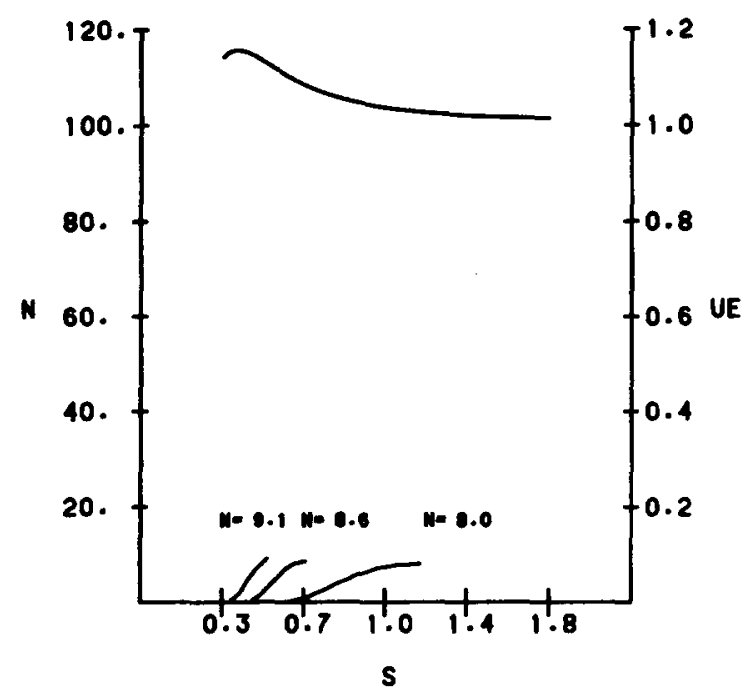

FIG. 9. Amplification-factor curves for different pressure-gradient situations on the body with optimized suction, $F=15.0 \times 10^{-6}$. Edge-speed ratio is shown as top curve.

order of magnitude higher than those for the two-dimensional problem where there is no pressure gradient. ${ }^{4}$ This implies that suction concentrated near branch $I$ in an adverse pressure gradient situation will dramatically decrease the maximum amplification factor as Figs. 4-7 indicate.

Other adverse pressure gradients are investigated by varying both the frequency and Reynolds number, thus moving all over the body. In our study we maintain the above constant dimensional volumetric flow rate per unit width of $4.73 \times 10^{-4} \mathrm{~m}^{3} / \mathrm{sec} / \mathrm{m}$ for the suction system.

First, a disturbance of constant frequency $F=15.0 \times 10^{-6}$ is subjected to three different pressure-gradient situations through adjustment of the unit Reynolds number. The disturbance in one instance experiences a favorable pressure-gradient and then an abrupt change to an unfavorable one, in another instance it undergoes a totally unfavorable pressure gradient including the maximum gradient for the body initially and in still a third instance it experiences a less adverse gradient. The results are shown in

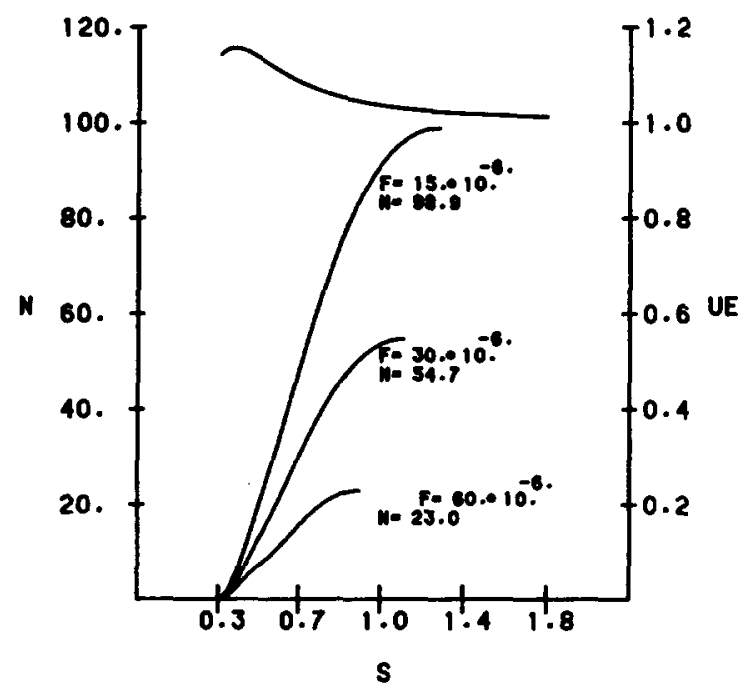

FIG. 10. Amplification-factor curves for the suctionless body for three different frequencies. Edge-speed ratio is shown as top curve.

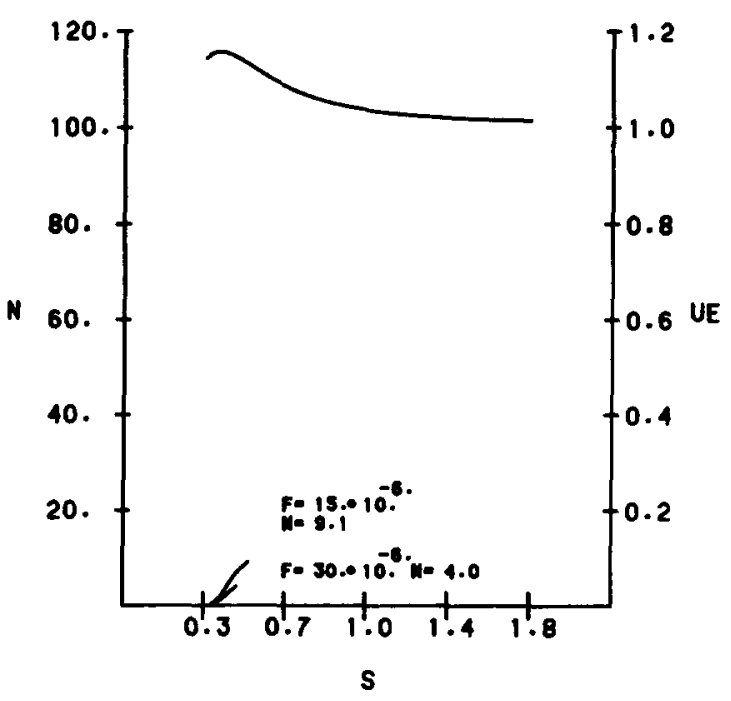

FIG. 11. Amplification-factor curves for optimized distributions of strips for three different frequencies. Edge-speed ratio is shown as top curve.

Figs. 8 and 9. Because of the destabilizing effect of an adverse gradient, in all three instances the disturbance grows significantly higher on the suctionless axisymmetric body than on the suctionless flat plate. For the flat plate the amplification factor attains a maximum value of 15.4. As seen in Fig. 8, the

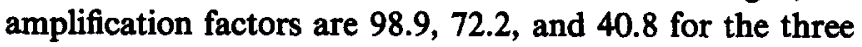
different cases mentioned above, respectively. In spite of the initial favorable gradient, the first case is associated with the largest amplification factors. The same adverse gradient of the second case exists in the first case, except that it occurs further downstream from branch I where the growth rates are greater. This accounts for the larger value of $N$. The third case has the smallest amplification factor because the adverse gradient is the least severe.

In Fig. 9 optimized suction distributions are applied to the body and the same three pressure-gradient distributions studied for $F=15.0 \times 10^{-6}$. In each case the suction reduces the amplification factors significantly and stabilization is complete in the latter half of the originally unstable, adverse

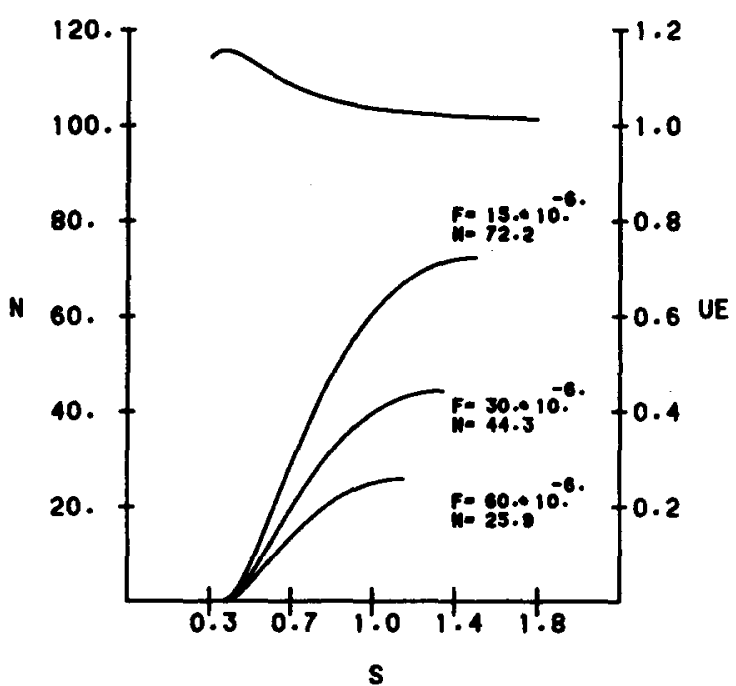

FIG. 12. Amplification-factor curves for the suctionless body for three different frequencies. Edge-speed ratio is shown as top curve. 


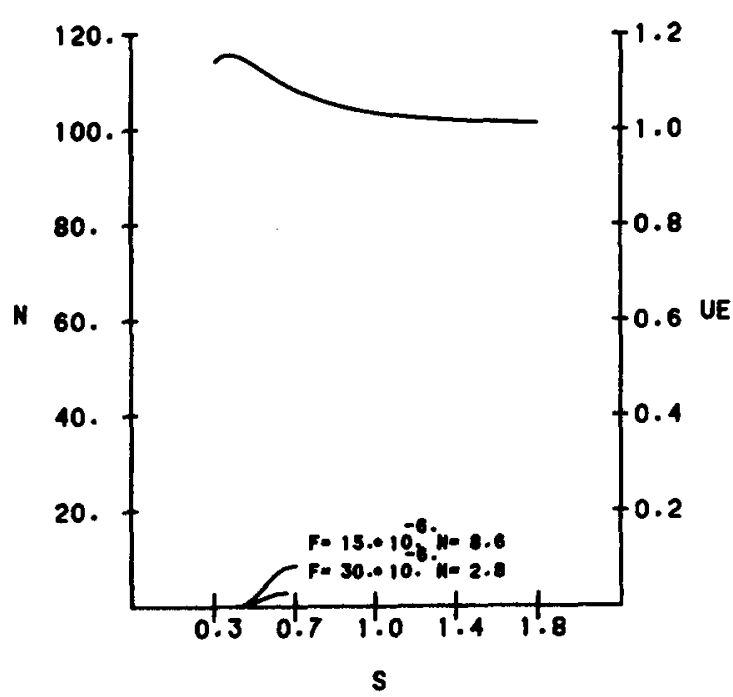

FIG. 13. Amplification-factor curves for optimized distributions of strips for three different frequencies. Edge-speed ratio is shown as top curve.

pressure-gradient regions. An interesting feature about Fig. 9 is that optimized suction results in about the same amplification factor for all three cases.

Next, disturbances of various frequencies are introduced into the boundary layer of the axisymmetric body for each individual pressure-gradient situation mentioned above. Three disturbance frequencies are considered: $F=15.0 \times 10^{-6}, 30.0 \times 10^{-6}$, and $60.0 \times 10^{-6}$. The corresponding unit Reynolds numbers are adjusted so that each disturbance is neutrally stable at the same initial point for each pressure-gradient situation. The results are shown in Figs. 10,12, and 14 for the suctionless body, and 11,13, and 15 for the body with optimized suction. In all cases the disturbance with the lowest frequency experiences the most growth; it is the most unstable.

Also, on the suctionless body, for each frequency the same comments as made above for a disturbance with

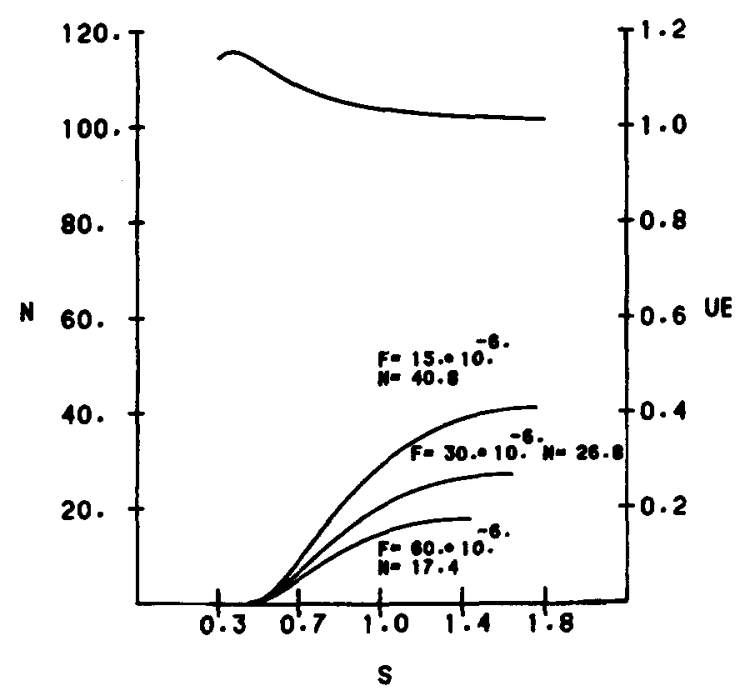

FIG. 14. Amplification-factor curves for the suctionless body for three different frequencies. Edge-speed ratio is shown as top curve.

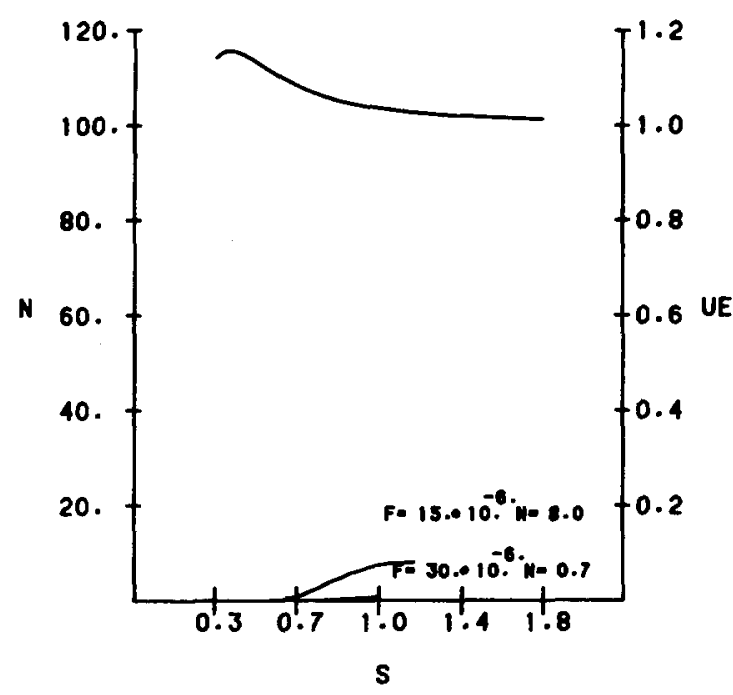

FIG. 15. Amplification-factor curves for optimized distributions of strips for three different frequencies. Edge-speed ratio is shown as top curve.

$F=15.0 \times 10^{-6}$ under various pressure-gradient conditions may be applied. The first situation where the disturbance initially experiences a favorable gradient and then an adverse gradient still accounts for the largest amplification factors for the two smaller frequencies. We believe that the pressure gradient is favorable for a period of growth that is slightly too long for the disturbance of $F=60.0 \times 10^{-6}$, resulting in the amplification factor for the first situation being slightly smaller than that for the second, i.e., 23.0 compared with 25.9. The third situation where the disturbance experiences the least adverse gradient still accounts for the lowest amplification factors for all frequencies.

When suction is optimized, the amplification factors again are reduced significantly and stabilization is complete in the latter half of the originally unstable region. In fact, the disturbance with $F=60.0 \times 10^{-6}$ is completely stabilized in all instances.

\section{ACKNOWLEDGMENTS}

This work was supported by the Fluid Dynamics Program of the Office of Naval Research under Contract No. N00014-85-K-0011, NR-061-201, and the Naval Underwater Sea Center through Westinghouse Electric Company.

\footnotetext{
'A. E. Gentry and A. R. Wazzan, David Taylor Naval Ship Research and Development Center, Code 1843 (1976).

${ }^{2}$ T. Cebeci and A. M. O. Smith, Analysis of Turbulent Boundary Layers (Academic, New York, 1974).

${ }^{3}$ H. B. Keller and T. Cebeci, AIAA J. 10, 1197 (1972).

${ }^{4}$ H. L. Reed and A. H. Nayfeh, submitted to AIAA J.

${ }^{5}$ G. A. Reynolds and W. S. Saric, submitted to AIAA J.

${ }^{6}$ F. M. White, Viscous Fluid Flow (McGraw-Hill, New York, 1974).

${ }^{7}$ K. Karamcheti, Principles of Ideal-Fluid Aerodynamics (Wiley, New York, 1966).

${ }^{8}$ H. L. Reed, Virginia Polytechnic Institute and State University, M. S. thesis, 1980.

${ }^{9}$ A. H. Nayfeh, H. L. Reed, and S. A. Ragab, submitted to Phys. Fluids.

${ }^{10}$ M. R. Scott and H. A. Watts, SIAM J. Num. Anal. 14, 40 (1977).

${ }^{11}$ N. A. Jaffe, J. J. Okamura, and A. M. O. Smith, AIAA J. 8, 301 (1970).

${ }^{12}$ A. H. Nayfeh, Introduction to Perturbation Techniques (Wiley-Interscience, New York, 1981).
} 